\title{
Vitamin D deficiency is associated with higher risks for SARS-CoV-2 infection and COVID-19 severity: a retrospective case-control study
}

\author{
Ariel Israel ${ }^{1} \cdot$ Assi Cicurel $^{1,2} \cdot$ Ilan Feldhamer ${ }^{1} \cdot$ Felicia Stern $^{3} \cdot$ Yosef Dror $^{3} \cdot$ Shmuel M. Giveon $^{4} \cdot$ David Gillis $^{5}$. \\ David Strich $^{6} \cdot$ Gil Lavie $^{1,7}$
}

Received: 17 September 2021 / Accepted: 20 November 2021 / Published online: 9 January 2022

(c) The Author(s), under exclusive licence to Società Italiana di Medicina Interna (SIMI) 2021

\begin{abstract}
Robust evidence of whether vitamin D deficiency is associated with COVID-19 infection and its severity is still lacking. The aim of the study was to evaluate the association between vitamin D levels and the risks of SARS-CoV-2 infection and severe disease in those infected. A retrospective study was carried out among members of Clalit Health Services (CHS), the largest healthcare organization in Israel, between March 1 and October 31, 2020. We created two matched case-control groups of individuals for which vitamin D levels and body mass index (BMI) were available before the pandemic: group (A), in which 41,757 individuals with positive SARS-CoV-2 PCR tests were matched with 417,570 control individuals without evidence of infection, and group (B), in which 2533 patients hospitalized in severe condition for COVID-19 were matched with 2533 patients who were tested positive for SARS-CoV-2, but were not hospitalized. Conditional logistic models were fitted in each of the groups to assess the association between vitamin D levels and outcome. An inverse correlation was demonstrated between the level of vitamin D and the risks of SARS-CoV-2 infection and of severe disease in those infected. Patients with very low vitamin D levels $(<30 \mathrm{nmol} / \mathrm{L})$ had the highest risks for SARS-CoV-2 infection and also for severe COVID-19 when infected-OR 1.246 [95\% CI 1.210-1.304] and 1.513 [95\% CI 1.230-1.861], respectively. In this large observational population study, we show a significant association between vitamin D deficiency and the risks of SARS-CoV-2 infection and of severe disease in those infected.
\end{abstract}

Keywords COVID-19 $\cdot$ SARS-CoV-2 $\cdot$ Vitamin D $\cdot$ Large population $\cdot$ Retrospective study

\section{Introduction}

Gil Lavie

gillav76@gmail.com; gillav@clalit.org.il

1 Branch of Planning and Strategy, Clalit Health Services, Tel Aviv, Israel

2 Southern District and Faculty of Health Sciences, Clalit Health Services, Ben-Gurion University of the Negev, Beersheba, Israel

3 School of Nutrition, Faculty of Agriculture, The Hebrew University of Jerusalem, Rehovot, Israel

4 Tel-Aviv University, Tel Aviv, Israel

5 Department of Pediatrics, Hadassah-Hebrew University Medical Center, Jerusalem, Israel

6 Pediatric Specialist Clinic, Clalit Health Services, Jerusalem District, Israel

7 Ruth and Bruce Rappaport Faculty of Medicine, Technion-Israel Institute of Technology, Haifa, Israel
The coronavirus disease 2019 (COVID-19) continues to spread around the globe since being declared a pandemic by the WHO in March 2020, causing significant morbidity and mortality. Due to the presence of the vitamin D receptor in various types of cells and tissue, vitamin D is known for its biologic activities on many organ systems and appears to play an essential role, as an immunomodulatory agent, in the prevention of respiratory infections $[1,2]$.

In light of its potential implications during sepsis, vitamin D may also have a potential role in COVID-19 pathophysiology, which has several clinical features in common with severe sepsis [2].

SARS-Cov-2 infection rate has been reported to be higher in countries with low vitamin $\mathrm{D}$ and in patients with low vitamin D $[4,6]$. Further research has demonstrated low vitamin D levels in patients with severe COVID-19 disease $[3,5]$. Nevertheless, observational studies so far 
demonstrated conflicting results and it is imperative to have more evidence based on large population-based studies to reveal the risk of COVID-19 in populations with vitamin D deficiency [25].

Clalit Health Services (CHS), the largest healthcare organization in Israel, provides comprehensive health services to over 4.7 million members and has centrally managed electronic health records (EHR) for over two decades, including laboratory tests, diagnoses, and hospitalization records [7]. This provides a unique opportunity to study the association between vitamin D levels and SARS-CoV-2 incidence and disease severity.

\section{Methods}

\section{Study population and data collection}

We collected from the CHS data warehouse selected variables from the EHR of individuals who underwent vitamin D testing between January 1, 2010, and February 29, 2020. For each individual, we extracted the last vitamin D level as well as the last BMI measured during the period. In addition, we collected the following demographic variables: date of birth (for age calculation), gender and 3-level socioeconomic status. We also extracted coded comorbidity diagnoses (cardiac arrhythmia, asthma, congestive heart failure (CHF), chronic obstructive pulmonary disease (COPD), chronic renal failure, diabetes mellitus, hypertension, ischemic heart disease (IHD), and malignancy). All baseline variables were collected on February 2020, before the onset of the pandemic. The primary care clinic was used to associate a geographic region, and one of the three main ethnic groups living in Israel, namely general, Ultra-Orthodox, and Arab.

We used the national database for COVID-19 established by the ministry of health to collect data from patients who had a positive RT-PCR test for SARS-CoV-2 and/or had been hospitalized with the disease between March 1- and October 31, 2020. We used these data to build two matched case-control groups. The first group (A) was designed to assess factors that affect the risk of SARS-CoV-2 infection: case patients were individuals with at least one positive SARS-CoV-2 test, with the date of the first positive test taken as the index date. As controls, for each SARS-CoV-2 positive patient we matched ten individuals without a positive SARS-CoV-2 test, and of the same age, gender, geographic region, and socioeconomic status, and assigned the same index date. A second group (B) was designed to assess factors that affect the risk of severe COVID-19 in patients with a positive test for SARS-CoV-2: case patients were individuals hospitalized in severe condition for COVID-19 (World Health Organization (WHO) severity scale [7] of 6 or above) or who died from the disease, with the date of the first positive test taken as the index date. As controls, for each patient hospitalized in severe condition we matched one individual with a positive SARS-CoV-2 test, and of the same age, gender, and socioeconomic status, but who was not hospitalized.

This study was approved by the CHS Institutional Review Board (IRB) with a waiver of informed consent.

\section{Laboratory and clinical measurements}

Vitamin D levels were tested by the LIAISON 25-OH vitamin D TOTAL assay (DiaSorin USA, Stillwater, Minn), a competitive 2-step chemiluminescence assay (67). The measuring range of this assay is 10 to $350 \mathrm{nmol} / \mathrm{L}$; analytical sensitivity is $<2.5 \mathrm{nmol} / \mathrm{L}$, and functional sensitivity is $<10.0 \mathrm{nmol} / \mathrm{L}$. The intra-assay precision is up to $5 \%$, and the inter-assay precision is up to $15 \%$. The specificity is $104 \%$ for $25-\mathrm{OH}$ vitamin D2 and $100 \%$ for $25-\mathrm{OH}$ vitamin D3. For patients with multiple vitamin $\mathrm{D}$ tests we used the last measured level during the study period. According to the measured levels, patients were assigned to four predefined vitamin $\mathrm{D}$ ranges (more than $75 \mathrm{nmol} / \mathrm{L}$-normal levels; $50-75 \mathrm{nmol} / \mathrm{L}$-insufficiency; $30-50 \mathrm{nmol} / \mathrm{L}$-deficiency, and less than $30 \mathrm{nmol} / \mathrm{L}$ - severe deficiency).

For each patient, we used the last BMI measurement documented in the EHR during patient encounters who took place before February 29, 2020. Patients were assigned to four predefined BMI categories (less than 25, 25-30, 30-35, and more than $35 \mathrm{~kg} / \mathrm{m}^{2}$ ) according to their last BMI measurement.

\section{Statistical analysis}

In descriptive tables, the statistical significance of differences observed between groups was assessed by the Fisher exact test for categorical variables, and the two-tailed Wilcoxon Mann-Whitney $U$ for continuous variables. Conditional logistic regression models were fitted for estimating the odds ratio [OR] and corresponding 95\% confidence interval [CI] of SARS-CoV-2 infection in matched group A and of severe COVID-19 disease in matched group B. In both groups, the association between vitamin $\mathrm{D}$ ranges and outcome was assessed first using univariable models, and then using several multivariable models, adjusted for ethnic group, BMI categories, and main comorbidities.

$p$ values below 0.05 were considered significant. Statistical analyses were performed using $\mathrm{R}$ statistical software version 3.6 ( $R$ Foundation for statistical computing).

\section{Results}

Between January 1, 2010, and February 29, 2020, $1,350,000$ distinct patients (about $30 \%$ of CHS members) had their serum vitamin D levels measured and these 
records were kept in CHS databases. From March 1 to October 31, 2020, 130,582 distinct CHS members had positive RT-PCR tests for SARS-Cov-2. Table 1 shows the comparison of the baseline characteristics of patients with positive tests (cases) vs. 4,502,455 CHS members without a positive test, who served as controls. The age and gender distribution of patients who were tested positive during this period were similar to the age and gender of the rest of the population, with a median age of 31-32 and about 51 percent of female individuals. Vitamin D levels observed in patients who were further tested positive were markedly lower: the median and interquartile range [IQR] of the last measured vitamin D levels were $47.8 \mathrm{nmol} / \mathrm{L}$ [31.4-65.2] for individuals who were further tested positive (cases) vs. $55.0 \mathrm{nmol} / \mathrm{L}$ [37.9-72.0] for controls $(p<0.001)$; severe vitamin $\mathrm{D}$ deficiency, defined by vitamin $\mathrm{D}$ levels below $30 \mathrm{nmol} / \mathrm{L}$, was present in $23.1 \%$ of case patients vs. $16.0 \%$ of control patients $(p<0.001)$. Marked differences could also be observed for the socioeconomic levels and ethnic groups affected by the disease. BMI, which was available for $95 \%$ of the patients, was slightly higher in cases, with a median BMI of $24.5 \mathrm{~kg} / \mathrm{m}^{2}$ for patients who were further tested positive vs. $23.5 \mathrm{~kg} / \mathrm{m}^{2}$ for controls.

Figure 1 displays the statistical distribution of baseline vitamin D levels among patients who were later tested positive for SARS-CoV-2 (red) vs. the rest of the population (gray) in males (top) and females (bottom). Lower vitamin $D$ levels were observed in patients who were later infected by SARS-CoV-2 $(p<0.001)$, and in particular, there was a large proportion of females with vitamin $\mathrm{D}$ values below $40 \mathrm{nmol} / \mathrm{L}$ among the cases. Previous studies in our health organization have shown that vitamin D deficiency is particularly prevalent in the Ultra-Orthodox Jewish and Arab population (particularly female). Remarkably, these two groups were disproportionately affected by SARS-CoV-2[8].

Having identified a high prevalence of vitamin D deficiency among SARS-CoV-2 patients, we proceeded to eliminate the possible effect of potential confounders by assessing the association between baseline vitamin $\mathrm{D}$ and the risk of SARS-CoV-2 infection, in a group matched for age, gender, geographical region, and socioeconomic status, with and without statistical adjustment for BMI, ethnic groups, and comorbidity. For this purpose, we built a matched group (A)
Table 1 Baseline characteristics of SARS-CoV-2-positive patients vs. other CHS members

\begin{tabular}{lll}
\hline & $\begin{array}{l}\text { SARS-CoV-2 positive } \\
N=130,582\end{array}$ & $\begin{array}{l}\text { Not SARS-CoV-2 positive } \\
N=4,502,455\end{array}$ \\
\hline Female (\%) & $67,084(51.4 \%)$ & $2,288,404(50.8 \%)$ \\
Age, median [IQR] & $32[17-50]$ & $31[12-51]$ \\
Socio Economic Status (\%) & & \\
Missing & $727(0.6 \%)$ & $23,687(0.5 \%)$ \\
Low & $67,605(51.8 \%)$ & $1,913,134(42.5 \%)$ \\
Medium & $45,781(35.1 \%)$ & $1,657,221(36.8 \%)$ \\
High & $16,469(12.6 \%)$ & $908,413(20.2 \%)$ \\
Ethnic group (\%) & & \\
General & $72,608(55.6 \%)$ & $3,046,183(67.7 \%)$ \\
Ultra-Orthodox & $20,348(15.6 \%)$ & $242,021(5.4 \%)$ \\
Arab Population & $37,626(28.8 \%)$ & $1,214,251(27.0 \%)$ \\
Vitamin D level last measured, median [IQR] & $47.8[31.4-65.2]$ & $55.0[37.9-72.0]$ \\
Patients with at least one measurement avail- & $42,977(32.9 \%)$ & $1,306,660(29.0 \%)$ \\
$\quad$ able during the period 2010-2019 & & \\
Range (\%) & & \\
$<30 \mathrm{nmol} / \mathrm{L}$ & $9925(23.1 \%)$ & $209,266(16.0 \%)$ \\
30-50 nmol/L & $13,134(30.6 \%)$ & $343,038(26.3 \%)$ \\
$50-75 \mathrm{nmol} / \mathrm{L}$ & $13,550(31.5 \%)$ & $474,173(36.3 \%)$ \\
$>75 \mathrm{nmol} / \mathrm{L}$ & $6368(14.8 \%)$ & $280,183(21.4 \%)$ \\
BMI, median [IQR] & $24.5[20.3-29.0]$ & $23.5[18.9-27.7]$ \\
Missing & $6237(4.8 \%)$ & $361,838(8.0 \%)$ \\
$<25 \mathrm{~kg} / \mathrm{m}^{2}$ & $66,136(53.2 \%)$ & $2,484,366(60.0 \%)$ \\
$25-30 \mathrm{~kg} / \mathrm{m}^{2}$ & $32,564(26.2 \%)$ & $998,231(24.1 \%)$ \\
30-35 kg/m & $17,027(13.7 \%)$ & $450,528(10.9 \%)$ \\
$>35 \mathrm{~kg} / \mathrm{m}^{2}$ & $8618(6.9 \%)$ & $207,492(5.0 \%)$ \\
\hline
\end{tabular}

$I Q R$ Interquartile Range, $B M I$ Body Mass Index 
Fig. 1 Distribution of vitamin D measured in the blood between years 2010-2020 among individuals later infected with SARS-CoV-2 and the rest of the population. Histograms showing the distribution of vitamin D levels measured in males (top) and females (bottom). The red histogram is for individuals who were further tested positive for SARS-CoV-2, in gray the rest of the population

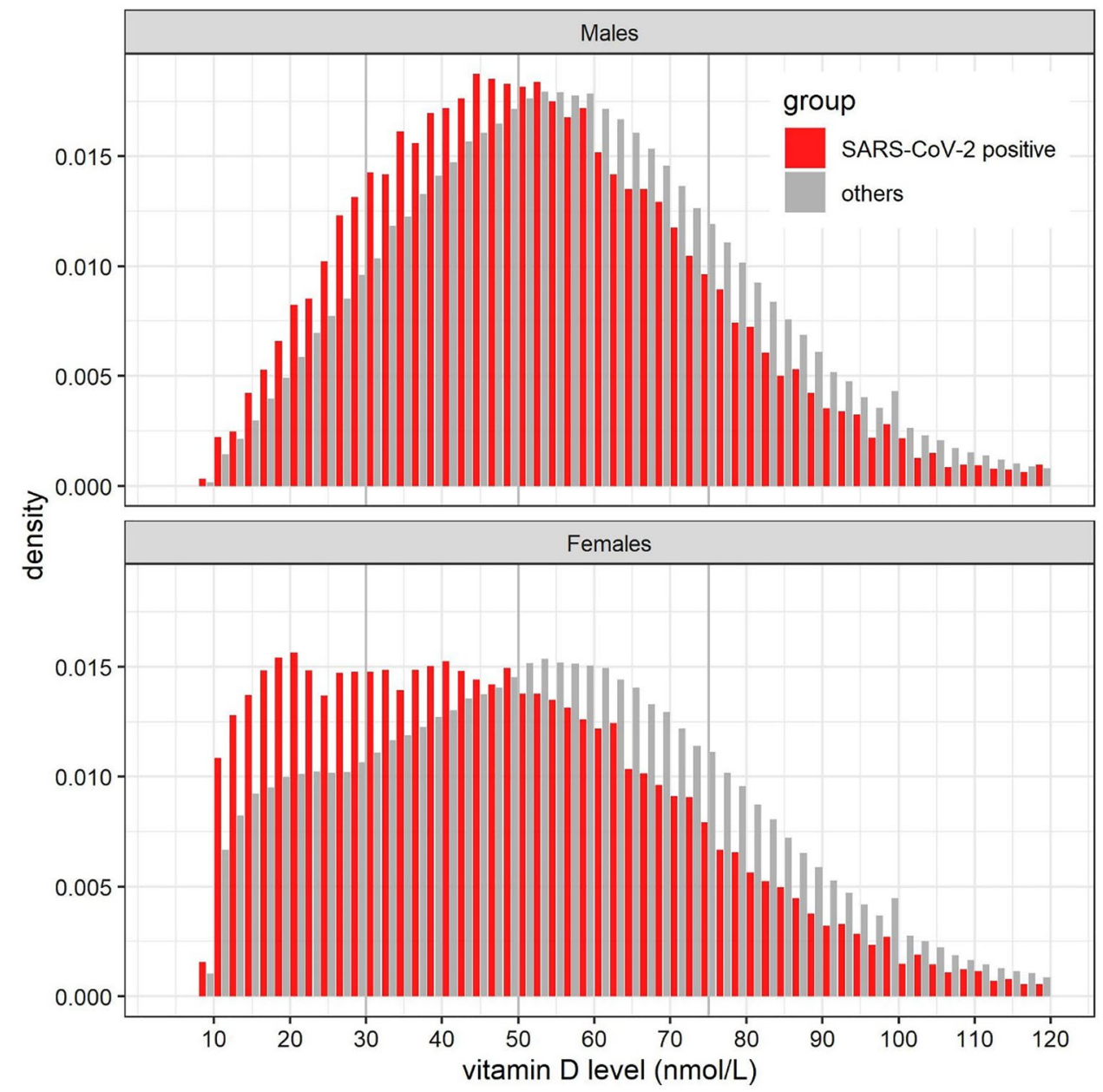

of patients who had their pre-pandemic vitamin D levels measured, where each patient who tested positive for SARSCoV-2 was matched to ten control patients of the same age, gender, geographic region, and socioeconomic status, as of February 2020, before the onset of the pandemics. A match was found for 41,757 individuals who were tested positive, with 417,570 assigned controls. The characteristics of the matched group are shown in Table 2. By design, the age, gender, regional, and socioeconomic status distribution of case and control patients were identical. The requirement of having prior vitamin $\mathrm{D}$ tests and the matching procedure increased the median age of this group (46 years), and the proportion of female individuals $(63.5 \%)$. Even after matching for age, gender, region, and socioeconomic status, marked differences subsisted in vitamin D levels, ethnic group, and BMI distributions between cases and controls.

Table 3 displays the conditional logistic regression results for SARS-CoV-2 infection status in the matched group. Model (1) is a univariate model based only on baseline vitamin $\mathrm{D}$ level ranges: compared to vitamin $\mathrm{D}$ levels above $75 \mathrm{nmol} / \mathrm{l}$, which served as reference value, severe vitamin $\mathrm{D}$ deficiency $(<30 \mathrm{nmol} / \mathrm{L})$ carried a higher risk of SARS-CoV-2 infection [OR $=1.442,95 \%$ CI 1.392-1.494], but even vitamin D insufficiency (between 50 and $75 \mathrm{nmol} / \mathrm{L}$ ) was associated with significantly increased risk. Model (2) is a multivariable model incorporating $\mathrm{BMI}$ in addition to baseline vitamin D levels. Compared with individuals with a BMI below $25 \mathrm{~kg} / \mathrm{m}^{2}$, overweight patients had markedly increased risk for SARS-CoV-2 infection, starting at BMI range 25-30 [OR $=1.198,95 \%$ CI 1.167-1.229], and the risk gradually increased for BMIs between 30 and 35 [OR $=1.407,95 \% \mathrm{CI} 1.365-1.451]$, and over 35 [OR $=1.471,95 \%$ CI 1.418-1.526]. An inverse correlation is frequently observed between serum vitamin $\mathrm{D}$ levels and BMI $[9,10]$, and lower vitamin D levels can be attributed to sequestration of vitamin D in fat tissues. In this model it appears that the association between vitamin $\mathrm{D}$ and SARS-CoV-2 infection risk is maintained even after adjustment for BMI. Model (3) is a multivariable model adjusted for ethnic group, in addition to BMI. Living in an ethnic group where there is high prevalence of SARS-CoV-2 infection incurs by itself a significantly increased risk, but even after controlling for these factors, vitamin D levels were associated with a significantly increased risk. Model 
Table 2 Demographics and clinical characteristics of matched group (A) of SARS-CoV-2-positive patients with matched controls

\begin{tabular}{|c|c|c|c|}
\hline & $\begin{array}{l}\text { Cases } \\
\text { SARS-CoV-2 positive } \\
N=41,757\end{array}$ & $\begin{array}{l}\text { Controls } \\
\text { not SARS-CoV-2 positive } \\
N=417,570\end{array}$ & $p$ value \\
\hline Female $(\%)$ & $26,508(63.5 \%)$ & $265,080(63.5 \%)$ & 1.000 \\
\hline Age, median [IQR] & $46[31-62]$ & $46[31-62]$ & 1.000 \\
\hline \multicolumn{4}{|l|}{ Socio Economic Status (\%) } \\
\hline Missing & $46(0.1 \%)$ & $460(0.1 \%)$ & 1.000 \\
\hline Low & $20,222(48.4 \%)$ & $202,220(48.4 \%)$ & 1.000 \\
\hline Medium & $15,335(36.7 \%)$ & $153,350(36.7 \%)$ & 1.000 \\
\hline High & $6154(14.7 \%)$ & $61,540(14.7 \%)$ & 1.000 \\
\hline \multicolumn{4}{|l|}{ Ethnic group (\%) } \\
\hline General & $23,163(55.5 \%)$ & $271,787(65.1 \%)$ & $<0.001$ \\
\hline Ultra-Orthodox & $7012(16.8 \%)$ & $41,195(9.9 \%)$ & $<0.001$ \\
\hline Arab Population & $11,582(27.7 \%)$ & $104,588(25.0 \%)$ & $<0.001$ \\
\hline Vitamin D level last measured, median [IQR] & $47.7[31.3,65.1]$ & $51.0[33.7,68.3]$ & $<0.001$ \\
\hline \multicolumn{4}{|l|}{ Range (\%) } \\
\hline$<30 \mathrm{nmol} / \mathrm{L}$ & $9740(23.3 \%)$ & $85,383(20.4 \%)$ & $<0.001$ \\
\hline $30-50 \mathrm{nmol} / \mathrm{L}$ & $12,735(30.5 \%)$ & $117,319(28.1 \%)$ & $<0.001$ \\
\hline $50-75 \mathrm{nmol} / \mathrm{L}$ & $13,130(31.4 \%)$ & $141,058(33.8 \%)$ & $<0.001$ \\
\hline$>75 \mathrm{nmol} / \mathrm{L}$ & $6152(14.7 \%)$ & $73,810(17.7 \%)$ & $<0.001$ \\
\hline BMI, median [IQR] & $26.6[22.9-30.9]$ & $25.7[22.3-29.8]$ & \\
\hline$<25 \mathrm{~kg} / \mathrm{m}^{2}$ & $16,298(39.0 \%)$ & $187,150(44.8 \%)$ & $<0.001$ \\
\hline $25-30 \mathrm{~kg} / \mathrm{m}^{2}$ & $13,060(31.3 \%)$ & $128,970(30.9 \%)$ & $<0.001$ \\
\hline $30-35 \mathrm{~kg} / \mathrm{m}^{2}$ & $7849(18.8 \%)$ & $65,593(15.7 \%)$ & $<0.001$ \\
\hline$>35 \mathrm{~kg} / \mathrm{m}^{2}$ & $4550(10.9 \%)$ & $35,857(8.6 \%)$ & $<0.001$ \\
\hline \multicolumn{4}{|l|}{ Comorbidity } \\
\hline Arrhythmia & $2522(6.0 \%)$ & $22,401(5.4 \%)$ & $<0.001$ \\
\hline Asthma & $2369(5.7 \%)$ & $23,934(5.7 \%)$ & 0.635 \\
\hline $\mathrm{CHF}$ & $1163(2.8 \%)$ & $9437(2.3 \%)$ & $<0.001$ \\
\hline COPD & $1011(2.4 \%)$ & $10,559(2.5 \%)$ & 0.185 \\
\hline Chronic Renal Failure & $1720(4.1 \%)$ & $14,395(3.4 \%)$ & $<0.001$ \\
\hline Diabetes Mellitus & $7467(17.9 \%)$ & $64,979(15.6 \%)$ & $<0.001$ \\
\hline Hypertension & $9848(23.6 \%)$ & $93,046(22.3 \%)$ & $<0.001$ \\
\hline IHD & $3367(8.1 \%)$ & $31,677(7.6 \%)$ & 0.001 \\
\hline Malignancy & $3228(7.7 \%)$ & $34,872(8.4 \%)$ & $<0.001$ \\
\hline \multicolumn{4}{|l|}{ COVID-19 disease severity } \\
\hline Not hospitalized for COVID-19 & $36,248(86.8 \%)$ & $417,570(100.0 \%)$ & \\
\hline Hospitalized in good condition & $2140(5.1 \%)$ & & \\
\hline Hospitalized in moderate condition & $818(2.0 \%)$ & & \\
\hline Hospitalized in severe condition & $1435(3.4 \%)$ & & \\
\hline Deceased & $1116(2.7 \%)$ & & \\
\hline
\end{tabular}

$I Q R$ Interquartile Range, $B M I$ Body Mass Index, $C H F$ Congestive Heart Failure, COPD Chronic Obstructive Pulmonary Disease, IHD Ischemic Heart Disease

(4) is a multivariable model adjusted for comorbidity, in addition to BMI and ethnic group. It appears that the risk of infection is slightly increased in patients with chronic renal failure $[\mathrm{OR}=1.154,95 \%$ CI 1.091-1.222], congestive heart failure (CHF) $[\mathrm{OR}=1.125,95 \%$ CI 1.048-1.208], diabetes mellitus [OR $=1.120,95 \%$ CI 1.086-1.156], and arrhythmia $[\mathrm{OR}=1.088,95 \%$ CI 1.03-1.143], while patients with malignancy appeared to have a slightly decreased risk of infection [OR $=0.932$ ], possibly reflecting increased adherence to social distancing among these patients. The association between vitamin $\mathrm{D}$ levels and infection risk persisted even after adjusting for these comorbidity factors.

After evaluating the association between vitamin D deficiency and SARS-CoV-2 infection status, we proceeded 
Table 3 Conditional logistic regression models for SARS-CoV-2 infection status in matched group (A) of SARS-CoV-2-positive patients with matched controls

\begin{tabular}{|c|c|c|c|c|c|c|c|c|}
\hline \multirow{4}{*}{$\begin{array}{l}\text { Model } \\
\text { Model Type } \\
\text { Explanatory vari- } \\
\text { ables }\end{array}$} & \multicolumn{2}{|l|}{ (1) } & \multicolumn{2}{|l|}{ (2) } & \multicolumn{2}{|l|}{ (3) } & \multicolumn{2}{|l|}{ (4) } \\
\hline & \multicolumn{2}{|l|}{ Univariable } & \multicolumn{6}{|l|}{ Multivariable } \\
\hline & \multicolumn{2}{|c|}{ vitamin $\mathrm{D}$ ranges } & \multicolumn{2}{|c|}{ vitamin $\mathrm{D}$ ranges + BMI } & \multicolumn{2}{|c|}{$\begin{array}{l}\text { vitamin D ranges + BMI } \\
\text { + ethnic group }\end{array}$} & \multicolumn{2}{|c|}{$\begin{array}{l}\text { vitamin D ranges }+ \text { BMI } \\
+ \text { ethnic group }+ \text { comorbidity }\end{array}$} \\
\hline & OR $(95 \% \mathrm{CI})$ & $p$ value & OR $(95 \% \mathrm{CI})$ & $p$ value & OR $(95 \% \mathrm{CI})$ & $p$ value & OR $(95 \% \mathrm{CI})$ & $p$ value \\
\hline \multicolumn{9}{|l|}{$\begin{array}{l}\text { Baseline vitamin D } \\
\text { range }\end{array}$} \\
\hline$<30 \mathrm{nmol} / \mathrm{L}$ & $\begin{array}{l}1.442(1.392- \\
1.494)\end{array}$ & $<0.001$ & $\begin{array}{l}1.364(1.316- \\
1.414)\end{array}$ & $<0.001$ & $\begin{array}{l}1.253(1.208- \\
1.301)\end{array}$ & $<0.001$ & $\begin{array}{l}1.246(1.210 \\
1.304)\end{array}$ & $<0.001$ \\
\hline $30-50 \mathrm{nmol} / \mathrm{L}$ & $\begin{array}{l}1.333(1.291- \\
1.377)\end{array}$ & $<0.001$ & $\begin{array}{l}1.272(1.231- \\
1.314)\end{array}$ & $<0.001$ & $\begin{array}{l}1.215(1.176- \\
1.256)\end{array}$ & $<0.001$ & $\begin{array}{l}1.211(1.177 \\
1.258)\end{array}$ & $<0.001$ \\
\hline $50-75 \mathrm{nmol} / \mathrm{L}$ & $\begin{array}{l}1.128(1.093- \\
1.164)\end{array}$ & $<0.001$ & $\begin{array}{l}1.099(1.065- \\
1.135)\end{array}$ & $<0.001$ & $\begin{array}{l}1.091(1.056- \\
1.126)\end{array}$ & $<0.001$ & $\begin{array}{l}1.091(1.056 \\
1.126)\end{array}$ & $<0.001$ \\
\hline$>75 \mathrm{nmol} / \mathrm{L}$ & Ref. & & Ref. & & Ref. & & Ref. & \\
\hline \multicolumn{9}{|l|}{ BMI category } \\
\hline$<25 \mathrm{~kg} / \mathrm{m}^{2}$ & & & Ref. & & Ref. & & Ref. & \\
\hline $25-30 \mathrm{~kg} / \mathrm{m}^{2}$ & & & $\begin{array}{l}1.198(1.167- \\
1.229)\end{array}$ & $<0.001$ & $\begin{array}{l}1.189(1.158- \\
1.220)\end{array}$ & $<0.001$ & $\begin{array}{l}1.182(1.152- \\
1.213)\end{array}$ & $<0.001$ \\
\hline $30-35 \mathrm{~kg} / \mathrm{m}^{2}$ & & & $\begin{array}{l}1.407(1.365- \\
1.451)\end{array}$ & $<0.001$ & $\begin{array}{l}1.384(1.343- \\
1.427)\end{array}$ & $<0.001$ & $\begin{array}{l}1.360(1.319- \\
1.403)\end{array}$ & $<0.001$ \\
\hline$>35 \mathrm{~kg} / \mathrm{m} 2$ & & & $\begin{array}{l}1.471(1.418- \\
1.526)\end{array}$ & $<0.001$ & $\begin{array}{l}1.449(1.397- \\
1.503)\end{array}$ & $<0.001$ & $\begin{array}{l}1.406(1.354- \\
1.460)\end{array}$ & $<0.001$ \\
\hline \multicolumn{9}{|l|}{ Ethnic group } \\
\hline $\begin{array}{l}\text { General popula- } \\
\text { tion }\end{array}$ & & & & & Ref. & & Ref. & \\
\hline Ultra-orthodox & & & & & $\begin{array}{l}2.834(2.732- \\
2.940)\end{array}$ & $<0.001$ & $\begin{array}{l}2.841(2.739- \\
2.948)\end{array}$ & $<0.001$ \\
\hline Arab & & & & & $\begin{array}{l}1.571(1.518- \\
1.625)\end{array}$ & $<0.001$ & $\begin{array}{l}1.558(1.505- \\
1.612)\end{array}$ & $<0.001$ \\
\hline \multicolumn{9}{|l|}{ Comorbidity } \\
\hline Arrhythmia & & & & & & & $\begin{array}{l}1.088(1.036- \\
1.143)\end{array}$ & 0.001 \\
\hline Asthma & & & & & & & $\begin{array}{l}1.007(0.963- \\
1.053)\end{array}$ & 0.753 \\
\hline $\mathrm{CHF}$ & & & & & & & $\begin{array}{l}1.125(1.048- \\
1.208)\end{array}$ & 0.002 \\
\hline COPD & & & & & & & $\begin{array}{l}0.929(0.867- \\
0.996)\end{array}$ & 0.038 \\
\hline $\begin{array}{l}\text { Chronic Renal } \\
\text { Failure }\end{array}$ & & & & & & & $\begin{array}{l}1.154(1.091- \\
1.222)\end{array}$ & $<0.001$ \\
\hline Diabetes Mellitus & & & & & & & $\begin{array}{l}1.120(1.086- \\
1.156)\end{array}$ & $<0.001$ \\
\hline Hypertension & & & & & & & $\begin{array}{l}1.010(0.978- \\
1.044)\end{array}$ & 0.544 \\
\hline IHD & & & & & & & $\begin{array}{l}0.988(0.944- \\
1.034)\end{array}$ & 0.599 \\
\hline Malignancy & & & & & & & $\begin{array}{l}0.932(0.896- \\
0.969)\end{array}$ & $<0.001$ \\
\hline
\end{tabular}

$O R$ odds ratio, $C I$ confidence Interval, $B M I$ body mass index, $C H F$ congestive heart failure, $C O P D$ chronic obstructive pulmonary disease, $I H D$ ischemic heart disease, ref. reference 
to investigate whether, among patients with evidence for SARS-CoV-2 infection, vitamin D levels were also associated with disease severity. For this purpose, we used the second matched group (B) where patients who were hospitalized with severe COVID-19 or who died from the disease and matched them 1:1 with control patients who had a positive SARS-CoV-2 PCR test but were not hospitalized for their disease. All patients in the matched group had baseline vitamin D levels, and controls were selected to match case patients' age, gender, and socioeconomic status. 2533 patients with severe disease were assigned 2533 matched controls. The characteristics of the matched group are presented in Table 4. By design, the age, gender, and socioeconomic status distribution of case and control patients are identical. The matching procedure increased the median age of the group to 74 years and the proportion of female individuals in the matched group is $51.8 \%$.

Table 5 displays conditional logistic regression results for severe COVID-19 infection or death in this second matched group. Model (1) shows that severe vitamin D
Table 4 Demographics and clinical characteristics of matched group (B) of COVID19 patients hospitalized in severe condition with matched controls of SARS-CoV2-positive individuals not hospitalized

\begin{tabular}{|c|c|c|c|}
\hline & $\begin{array}{l}\text { Cases } \\
\text { COVID-19 severe } \\
N=2533\end{array}$ & $\begin{array}{l}\text { Controls } \\
\text { SARS-CoV-2 positive } \\
\text { not hospitalized } N=2533\end{array}$ & $p$ value \\
\hline Female (\%) & $1313(51.8 \%)$ & $1313(51.8 \%)$ & 1.000 \\
\hline Age, median [IQR] & $74[63-83]$ & $74[63-83]$ & 1.000 \\
\hline \multicolumn{4}{|l|}{ Socio Economic Status (\%) } \\
\hline Missing & $301(11.9 \%)$ & $301(11.9 \%)$ & 1.000 \\
\hline Low & $1091(43.1 \%)$ & $1091(43.1 \%)$ & 1.000 \\
\hline Medium & $1102(43.5 \%)$ & $1102(43.5 \%)$ & 1.000 \\
\hline High & $39(1.5 \%)$ & $39(1.5 \%)$ & 1.000 \\
\hline \multicolumn{4}{|l|}{ Ethnic group (\%) } \\
\hline General & $1647(65.0 \%)$ & $1774(70.0 \%)$ & $<0.001$ \\
\hline Ultra-Orthodox & $201(7.9 \%)$ & $311(12.3 \%)$ & $<0.001$ \\
\hline Arab Population & $685(27.0 \%)$ & $448(17.7 \%)$ & $<0.001$ \\
\hline Vitamin D level last measured, median [IQR] & $47.8[30.9,67.0]$ & $53.8[36.9,71.1]$ & $<0.001$ \\
\hline \multicolumn{4}{|l|}{ Range $(\%)$} \\
\hline$<30 \mathrm{nmol} / \mathrm{L}$ & $613(24.2 \%)$ & $425(16.8 \%)$ & $<0.001$ \\
\hline $30-50 \mathrm{nmol} / \mathrm{L}$ & $720(28.4 \%)$ & $706(27.9 \%)$ & 0.685 \\
\hline $50-75 \mathrm{nmol} / \mathrm{L}$ & $777(30.7 \%)$ & $893(35.3 \%)$ & $<0.001$ \\
\hline$>75 \mathrm{nmol} / \mathrm{L}$ & $423(16.7 \%)$ & $509(20.1 \%)$ & $<0.001$ \\
\hline BMI, median [IQR] & $29.1[25.5-33.6]$ & 27.9 [24.9-31.6] & $<0.001$ \\
\hline$<25 \mathrm{~kg} / \mathrm{m}^{2}$ & $560(22.1 \%)$ & $654(25.8 \%)$ & 0.002 \\
\hline $25-30 \mathrm{~kg} / \mathrm{m}^{2}$ & $853(33.7 \%)$ & $999(39.4 \%)$ & $<0.001$ \\
\hline $30-35 \mathrm{~kg} / \mathrm{m}^{2}$ & $642(25.3 \%)$ & $573(22.6 \%)$ & 0.025 \\
\hline$>35 \mathrm{~kg} / \mathrm{m}^{2}$ & $478(18.9 \%)$ & $307(12.1 \%)$ & $<0.001$ \\
\hline \multicolumn{4}{|l|}{ Comorbidity } \\
\hline Arrhythmia & $591(23.3 \%)$ & $446(17.6 \%)$ & $<0.001$ \\
\hline Asthma & $203(8.0 \%)$ & $156(6.2 \%)$ & 0.010 \\
\hline $\mathrm{CHF}$ & $428(16.9 \%)$ & $172(6.8 \%)$ & $<0.001$ \\
\hline COPD & $278(11.0 \%)$ & $141(5.6 \%)$ & 0.185 \\
\hline Chronic Renal Failure & $581(22.9 \%)$ & $240(9.5 \%)$ & $<0.001$ \\
\hline Diabetes Mellitus & $1308(51.6 \%)$ & $896(35.4 \%)$ & $<0.001$ \\
\hline Hypertension & $1748(69.0 \%)$ & $1440(56.8 \%)$ & $<0.001$ \\
\hline IHD & $785(31.0 \%)$ & $571(22.5 \%)$ & $<0.001$ \\
\hline Malignancy & $478(18.9 \%)$ & $389(15.4 \%)$ & 0.001 \\
\hline \multicolumn{4}{|l|}{ COVID-19 disease severity } \\
\hline Not hospitalized & & $2533(100.0 \%)$ & \\
\hline Hospitalized in severe condition & $1430(56.5 \%)$ & & \\
\hline Deceased & $1103(43.5 \%)$ & & \\
\hline
\end{tabular}

$I Q R$ interquartile range, $B M I$ body mass index, $C H F$ congestive heart failure, $C O P D$ chronic obstructive pulmonary disease, $I H D$ ischemic heart disease 
deficiency $(<30 \mathrm{nmol} / \mathrm{L})$ carries a significantly increased risk for hospitalization with severe disease $[\mathrm{OR}=1.777$, 95\% CI 1.477-2.138], and this risk was also increased for $30-50 \mathrm{nmol} / \mathrm{L}$ [OR $=1.256,95 \%$ CI $1.057-1.492]$. The association between low vitamin D levels and severe disease is maintained in multivariable models adjusted for BMI (2), ethnic group (3), and comorbidity factors (4). We observed a significantly increased risk of severe disease for infected patients with chronic renal failure $[\mathrm{OR}=2.077,95 \%$ CI $1.721-2.506]$, CHF $[\mathrm{OR}=1.757$, 95\% CI 1.404-2.198], Chronic Obstructive Pulmonary Disease (COPD) [OR $=1.511,95 \%$ CI 1.172-1.947], diabetes mellitus [OR $=1.491,95 \%$ CI 1.300-1.709], malignancy $[\mathrm{OR}=1.343,95 \% \mathrm{CI} 1.134-1.590]$, and hypertension $[\mathrm{OR}=1.280,95 \%$ CI $1.097-1.494]$. The association between vitamin $\mathrm{D}$ levels and infection risk is maintained even after adjustment for comorbidity factors. Even in the fully adjusted model [4], there was a significantly increased risk for severe disease for patients with vitamin $\mathrm{D}$ below $30 \mathrm{nmol} / \mathrm{L}[\mathrm{OR}=1.513,95 \% \mathrm{CI} 1.230-1.861]$, and between 30 and $50 \mathrm{nmol} / \mathrm{L}$ [OR $=1.311,95 \%$ CI $1.083-1.587]$.

\section{Discussion}

In this large retrospective case-control study, an inverse correlation was demonstrated between the baseline level of vitamin D and the risks of SARS-CoV-2 infection and of severe COVID-19 disease when infected. Significant associations were also found between obesity and comorbidity factors and the studied outcomes. However, even after adjusting for these factors, low vitamin D levels remained significantly associated with the outcomes.

Our report confirms the results of several other observational studies showing association between low vitamin D levels and COVID-19, particularly those suffering from severe disease $[4,12,13,21-23,26]$. In addition, northern latitude (associated with lower vitamin D levels) was found to be associated with a higher hospitalization rate for COVID-19 as well as a higher mortality rate compared with Southern latitudes [2]. Nevertheless, a cohort of workingage adults found no evidence for an independent association between low levels of vitamin D and SARS-CoV-2 seropositivity [20], and recent review concluded that there is currently insufficient information to guide the use of vitamin D as a treatment for COVID-19, as the evidence for the effectiveness of vitamin D supplementation for the treatment of COVID-19 is uncertain, and there is only limited safety information. [24].

Several potential mechanisms may explain the observed association between vitamin D levels and SARS-CoV-2 incidence and disease severity [14]. Notably, respiratory viruses disrupt cell junction integrity [15], while vitamin D maintains cell junctions and exhibits protective effects against endothelial dysfunction and thrombosis. Furthermore, vitamin D enhances cellular innate immunity partly through the induction of antimicrobial peptides which may interfere with viral replication [16].

Our study has several unique strengths: a very large population of individuals with vitamin $D$ levels measured in community settings before the pandemic; the accurate BMI data, as proper documentation of BMI during patient encounters was evaluated as a quality measure in our health organization; the extensive demographic and clinical data documented in the electronic health records allowing for adequate data control; and the ability to reliably identify all patients with positive SARS-CoV-2 testing and the COVID-19 hospitalization outcomes, thanks to a central database established by the Israeli Ministry of Health, and the universal use of the national ID number as patient identifier. Although having a comprehensive demographic and clinical background data, we acknowledge our study's limitations as being observational, noting the difficulty in eliminating all possible confounders. Whether vitamin D plays a causal role in COVID-19 pathophysiology or just a marker of ill health is not known, and our results should be carefully interpreted, as patients positive for SARSCoV-2 and with severe COVID-19 had a higher number of comorbidities.

A major limitation of our study is the long time range during which vitamin D levels were measured before eventual infection or hospitalization, and lack of information on treatment with vitamin D supplements during this period. We hypothesized that patients with low levels of vitamin $\mathrm{D}$ who were treated with supplements performed a repeat test to monitor its level, as a blood test is recommended to monitor blood levels few months after beginning treatment [25]. We therefore extracted for each individual the latest vitamin $D$ level available.

Interventional, randomized controlled trials are classically required to establish causality of observed statistical associations. Small clinical trials have shown promising results [17, 18], and other bigger trials are underway [19]. In the context of a rapidly spreading pandemic with a high casualty rate, the increasing body of evidence showing significantly increased risk in vitamin D deficient patients on one hand, and the relative known safety of daily vitamin D intake at recommended doses on the other hand, one may argue in favor of maintaining normal levels of vitamin D as a prevention measure, in particular for populations at risk [27]. Further large randomized controlled trials are warranted to determine if vitamin $\mathrm{D}$ supplementation can decrease COVID-19 incidence and its severity. 
Table 5 Conditional logistic regression models for severe disease or death due to COVID-19, in matched group (B)

\begin{tabular}{|c|c|c|c|c|c|c|c|c|}
\hline \multirow{4}{*}{$\begin{array}{l}\text { Model } \\
\text { Model type } \\
\text { Explanatory vari- } \\
\text { ables }\end{array}$} & \multirow{2}{*}{\multicolumn{2}{|c|}{$\frac{(1)}{\text { Univariable }}$}} & \multicolumn{2}{|l|}{ (2) } & \multicolumn{2}{|l|}{ (3) } & \multicolumn{2}{|l|}{ (4) } \\
\hline & & & \multicolumn{6}{|l|}{ Multivariable } \\
\hline & \multicolumn{2}{|c|}{ vitamin $\mathrm{D}$ ranges } & \multicolumn{2}{|c|}{ vitamin D ranges + BMI } & \multicolumn{2}{|c|}{$\begin{array}{l}\text { vitamin D ranges + BMI } \\
\text { + ethnic group }\end{array}$} & \multicolumn{2}{|c|}{$\begin{array}{l}\text { vitamin D ranges + BMI } \\
+ \text { ethnic group }+ \text { comorbidit }\end{array}$} \\
\hline & OR $(95 \% \mathrm{CI})$ & $p$ value & OR $(95 \% \mathrm{CI})$ & $p$ value & OR $(95 \% \mathrm{CI})$ & $p$ value & OR $(95 \% \mathrm{CI})$ & $p$ value \\
\hline \multicolumn{9}{|l|}{$\begin{array}{l}\text { Baseline vitamin } \mathrm{D} \\
\text { range }\end{array}$} \\
\hline$<30 \mathrm{nmol} / \mathrm{L}$ & $\begin{array}{l}1.777(1.477- \\
2.138)\end{array}$ & $<0.001$ & $\begin{array}{l}1.629(1.349- \\
1.966)\end{array}$ & $<0.001$ & $\begin{array}{l}1.483(1.223- \\
1.799)\end{array}$ & $<0.001$ & $\begin{array}{l}1.513(1.230- \\
1.861)\end{array}$ & $<0.001$ \\
\hline $30-50 \mathrm{nmol} / \mathrm{L}$ & $\begin{array}{l}1.256(1.057- \\
1.492)\end{array}$ & 0.010 & $\begin{array}{l}1.177(0.988- \\
1.403)\end{array}$ & 0.069 & $\begin{array}{l}1.175(0.984- \\
1.403)\end{array}$ & 0.075 & $\begin{array}{l}1.311(1.083- \\
1.587)\end{array}$ & 0.006 \\
\hline $50-75 \mathrm{nmol} / \mathrm{L}$ & $\begin{array}{l}1.058(0.863- \\
1.208)\end{array}$ & 0.504 & $\begin{array}{l}1.021(0.863- \\
1.208)\end{array}$ & 0.806 & $\begin{array}{l}1.033(0.872- \\
1.224)\end{array}$ & 0.705 & $\begin{array}{l}1.127(0.940- \\
1.351)\end{array}$ & 0.196 \\
\hline$>75 \mathrm{nmol} / \mathrm{L}$ & Ref. & & Ref. & & Ref. & & Ref. & \\
\hline \multicolumn{9}{|l|}{ BMI category } \\
\hline$<25 \mathrm{~kg} / \mathrm{m}^{2}$ & & & Ref. & & Ref. & & Ref. & \\
\hline $25-30 \mathrm{~kg} / \mathrm{m}^{2}$ & & & $\begin{array}{l}0.986(0.848- \\
1.147)\end{array}$ & 0.859 & $0.97(0.833-1.129)$ & 0.691 & $\begin{array}{l}0.892(0.757- \\
1.050)\end{array}$ & 0.170 \\
\hline $30-35 \mathrm{~kg} / \mathrm{m}^{2}$ & & & $\begin{array}{l}1.274(1.077- \\
1.506)\end{array}$ & 0.005 & $\begin{array}{l}1.247(1.052- \\
1.477)\end{array}$ & 0.011 & $\begin{array}{l}1.127(0.938- \\
1.354)\end{array}$ & 0.204 \\
\hline$>35 \mathrm{~kg} / \mathrm{m} 2$ & & & $\begin{array}{l}1.784(1.469- \\
2.167)\end{array}$ & $<0.001$ & $\begin{array}{l}1.712(1.406- \\
2.086)\end{array}$ & $<0.001$ & $1.34(1.083-1.659)$ & 0.008 \\
\hline \multicolumn{9}{|l|}{ Ethnic group } \\
\hline $\begin{array}{l}\text { General popula- } \\
\text { tion }\end{array}$ & & & & & Ref. & & Ref. & \\
\hline Ultra-orthodox & & & & & $\begin{array}{l}0.682(0.554- \\
0.839)\end{array}$ & $<0.001$ & $\begin{array}{l}0.802(0.643- \\
1.000)\end{array}$ & 0.051 \\
\hline Arab & & & & & $\begin{array}{l}1.569(1.334- \\
1.845)\end{array}$ & $<0.001$ & $\begin{array}{l}1.470(1.238- \\
1.746)\end{array}$ & $<0.001$ \\
\hline \multicolumn{9}{|l|}{ Comorbidity } \\
\hline Arrhythmia & & & & & & & $\begin{array}{l}1.037(0.874- \\
1.231)\end{array}$ & 0.679 \\
\hline Asthma & & & & & & & $\begin{array}{l}1.133(0.884- \\
1.452)\end{array}$ & 0.325 \\
\hline $\mathrm{CHF}$ & & & & & & & $\begin{array}{l}1.757(1.404- \\
2.198)\end{array}$ & $<0.001$ \\
\hline COPD & & & & & & & $\begin{array}{l}1.511(1.172- \\
1.947)\end{array}$ & 0.002 \\
\hline $\begin{array}{l}\text { Chronic Renal } \\
\text { Failure }\end{array}$ & & & & & & & $\begin{array}{l}2.077(1.721- \\
2.506)\end{array}$ & $<0.001$ \\
\hline Diabetes Mellitus & & & & & & & $\begin{array}{l}1.491(1.300- \\
1.709)\end{array}$ & $<0.001$ \\
\hline Hypertension & & & & & & & $\begin{array}{l}1.280(1.097- \\
1.494)\end{array}$ & 0.002 \\
\hline IHD & & & & & & & $\begin{array}{l}1.089(0.930- \\
1.274)\end{array}$ & 0.291 \\
\hline Malignancy & & & & & & & $\begin{array}{l}1.343(1.134- \\
1.590)\end{array}$ & 0.001 \\
\hline
\end{tabular}

$O R$ odds ratio, $C I$ confidence interval, $B M I$ body mass index, $C H F$ congestive heart failure, $C O P D$ chronic obstructive pulmonary disease, $I H D$ ischemic heart disease, $r e f$. reference 


\section{Conclusion}

In this large observational population study, we show a significant association between vitamin D deficiency and the risks of SARS-CoV-2 infection and of severe disease in those infected.

Author contributions All authors contributed to the study conception and design. Material preparation, data collection, and analysis were performed by Ariel Israel and Ilan Feldhamer. The first draft of the manuscript was written by Ariel Israel and Gil Lavie and all the authors commented on previous versions of the manuscript. All the authors read and approved the final manuscript.

\section{Funding None.}

Availability of data and material The datasets generated during and/or analyzed during the current study are available from the corresponding author on reasonable request.

\section{Declarations}

\section{Conflict of interest None.}

Human and animal rights statement and Informed consent This study was approved by the CHS Institutional Review Board (IRB) with a waiver of informed consent due to the anonymous nature of the data, approval number: COM-0046-20.

Consent to participate Not applicable.

Consent for publication Not applicable.

\section{References}

1. Holick MF (2007) Medical progress: vitamin D deficiency. N Engl J Med 357(3):266-281

2. Rossetti M, Martucci G, Starchl C, Amrein K (2021) Micronutrients in Sepsis and COVID-19: a narrative review on what we have learned and what we want to know in future trials. Medicina 57(5):419

3. Rhodes JM, Subramanian S, Laird E, Kenny RA (2020) Editorial: low population mortality from COVID-19 in countries south of latitude 35 degrees North supports vitamin D as a factor determining severity. Aliment Pharmacol Ther 51(12):1434-1437

4. Merzon E, Tworowski D, Gorohovski A, Vinker S, Golan Cohen A, Green I, Frenkel-Morgenstern M (2020) Low plasma 25(OH) vitamin D level is associated with increased risk of COVID-19 infection: an Israeli population-based study. FEBS J 13:644

5. Panagiotou G, Tee SA, Ihsan Y, Athar W, Marchitelli G, Kelly D, Boot CS, Stock N, Macfarlane J, Martineau AR, Burns G, Quinton R (2020) Low serum 25-hydroxyvitamin D (25[OH]D) levels in patients hospitalised with COVID-19 are associated with greater disease severity. Clin Endocrinol. https://doi.org/10.1111/ cen. 14276
6. D'Avolio A, Avataneo V, Manca A et al (2020) 25-hydroxyvitamin D concentrations are lower in patients with positive PCR for SARS-CoV-2. Nutrients 12:1359

7. Dror Y, Giveon SM, Hoshen M, Feldhamer I, Balicer RD, Feldman BS (2013) Vitamin D levels for preventing acute coronary syndrome and mortality: evidence of a nonlinear association. $\mathrm{J}$ Clin Endocrinol Metab 98(5):2160-2167

8. Marshall JC, Murthy S, Diaz J, Adhikari N, Angus DC, Arabi YM, Baillie K, Bauer M, Berry S, Blackwood B, Bonten M, Bozza F, Brunkhorst F, Cheng A, Clarke M, Dat VQ, de Jong M, Denholm J, Derde L, Dunning J, Feng X, Fletcher T, Foster N, Fowler R, Gobat N, Gomersall C, Gordon A, Glueck T, Harhay M, Hodgson C, Horby P, Kim YJ, Kojan R, Kumar B, Laffey J, Malvey D, Martin-Loeches I, McArthur C, McAuley D, McBride S, McGuinness S, Merson L, Morpeth S, Needham D, Netea M, Oh MD, Phyu S, Piva S, Qiu R, Salisu-Kabara H, Shi L, Shimizu N, Sinclair J, Tong S, Turgeon A, Uyeki T, van de Veerdonk F, Webb S, Williamson P, Wolf T, Zhang J (2020) A minimal common outcome measure set for COVID-19 clinical research. Lancet Infect Dis 20(8):e192-e197

9. Israel A, Cicurel A, Feldhamer I, Yosef Dror B, Giveon SM, Gillis D, Strich D, Lavie G (2020) The link between vitamin D deficiency and Covid-19 in a large population. medRxiv. https://doi. org/10.1101/2020.10.13.20211953

10. Kremer R, Campbell PP, Reinhardt T, Gilsanz V (2009) Vitamin $\mathrm{D}$ status and its relationship to body fat, final height, and peak bone mass in young women. J Clin Endocrinol Metab 94(1):67-73

11. Carter SJ, Baranauskas MN, Fly AD (2020) Considerations for obesity, vitamin $\mathrm{D}$, and physical activity amid the COVID-19 pandemic. Obesity 28(7):1176-1177

12. Meltzer DO, Best TJ, Zhang H, Vokes T, Arora V, Solway J (2020) Association of vitamin D status and other clinical characteristics with COVID-19 test results. JAMA Netw Open 3(9):e2019722

13. De Smet D, De Smet K, Herroelen P, Gryspeerdt S, Martens GA (2020) Serum 25(OH)D level on hospital admission associated with COVID-19 stage and mortality. Am J Clin Pathol. https:// doi.org/10.1093/ajcp/aqaa252

14. Schwalfenberg GK (2011) A review of the critical role of vitamin $\mathrm{D}$ in the functioning of the immune system and the clinical implications of vitamin D deficiency. Mol Nutr Food Res 55(1):96-108

15. Zhang Y, Wu S, Sun J, Vitamin D (2013) vitamin D receptor and tissue barriers. Tissue Barriers 1(1):e23118

16. Gil Á, Plaza-Diaz J, Mesa MD (2018) Vitamin D: classic and novel actions. Ann Nutr Metab 72(2):87-95

17. Quesada-Gomez JM, Entrenas-Castillo M, Bouillon R (2020) Vitamin $\mathrm{D}$ receptor stimulation to reduce acute respiratory distress syndrome (ARDS) in patients with coronavirus SARS-CoV-2 infections: Revised Ms SBMB 2020_166. J Steroid Biochem Mol Biol. https://doi.org/10.1016/j.jsbmb.2020.105719

18. Annweiler C, Hanotte B, Grandin de l'Eprevier C, Sabatier JM, Lafaie L, Célarier T (2020) Vitamin D and survival in COVID-19 patients: a quasi-experimental study. J Steroid Biochem Mol Biol 204:105771

19. Vitamin D | COVID-19-list results-ClinicalTrials.gov. (2021) Available at: https://clinicaltrials.gov/ct2/results?cond=COVID$19 \&$ term $=$ vitamin $+D \&$ cntry $=\&$ state $=\&$ city $=\&$ dist $=$. Accessed 20 Dec 2020

20. Li Y, Tong CH, Bare LA, Devlin JJ (2021) Assessment of the association of vitamin D level with SARS-CoV-2 seropositivity among working-age adults. JAMA Netw Open 4(5):e2111634

21. Teshome A, Adane A, Girma B, Mekonnen ZA (2021) The impact of vitamin D level on COVID-19 infection: systematic review and meta-analysis. Front Public Health 9:624559

22. Nimavat N, Singh S, Singh P, Singh SK, Sinha N (2021) Vitamin D deficiency and COVID-19: a case-control study at a tertiary care hospital in India. Ann Med Surg 68:102661 
23. Wang Z, Joshi A, Leopold K, Jackson S, Christensen S, Nayfeh T, Mohammed K, Creo A, Tebben P, Kumar S (2021) Association of vitamin D deficiency with COVID-19 infection severity: systematic review and meta-analysis. Clin Endocrinol. https://doi. org/10.1111/cen.14540

24. Stroehlein JK, Wallqvist J, Iannizzi C, Mikolajewska A, Metzendorf MI, Benstoem C, Meybohm P, Becke M, Skoetz N, Stegemann M, Piechotta V (2021) Vitamin D supplementation for the treatment of COVID-19: a living systematic review. Cochrane Database Syst Rev. https://doi.org/10.1002/14651858.CD015043

25. https://www.uptodate.com/contents/vitamin-d-deficiency-beyondthe-basics. Accessed 6 July 2021

26. Jude EB, Ling SF, Allcock R, Yeap BX, Pappachan JM (2021) Vitamin D deficiency is associated with higher hospitalization risk from COVID-19: a retrospective case-control study. J Clin Endocrinol Metab. https://doi.org/10.1210/clinem/dgab439

27. Mirijello A, D'Errico MM, La Marca A, Piscitelli P, De CosmoComment $S$ et al (2020) Comment on Matricardi et al. Pediatr Allergy Immunol 31(8):997

Publisher's Note Springer Nature remains neutral with regard to jurisdictional claims in published maps and institutional affiliations. 